\title{
A New Line-Shape Asymmetry Model for Wavelength Modulation Spectroscopy in Gaseous Flows
}

\author{
Václav Nevrlý1 ${ }^{1}$, Vít Klečka ${ }^{1}$, Michal Vašinek ${ }^{2}$, Václav Válek ${ }^{1}$, Jan Suchánek ${ }^{3}$, Michal Dostál ${ }^{1,3}$ \\ Branislav Hric ${ }^{1}$, Petr Bitala ${ }^{1}$ and Zdeněk Zelinger ${ }^{3}$ \\ ${ }^{1}$ Faculty of Safety Engineering, VŠB-Technical University of Ostrava, Lumírova, 13, 700 30, Ostrava-Výškovice, Czech Re- \\ public, vaclav.nevrly@vsb.cz \\ ${ }^{2}$ Faculty of Electrical Engineering and Computer Science, VŠB-Technical University of Ostrava, 17. listopadu, 15, 708 33, \\ Ostrava-Poruba, Czech Republic \\ ${ }^{3} J$. Heyrovský Institute of Physical Chemistry, The Czech Academy of Sciences, Dolejškova, 2155/3, 182 23, Prague, Czech \\ Republic
}

This communication reports technical notes on the development and application of an automated line-shape fitting procedure for wavelength modulation spectroscopy (WMS). Near-infrared transitions of carbon dioxide $\left(\mathrm{CO}_{2}\right)$ around $1573 \mathrm{~nm}$ were measured in vertical cold (nonreacting) flow of $\mathrm{CO}_{2}$ at atmospheric pressure using WMS with demodulation at second harmonic frequency. Semi-empirical model based on the set of so-called Gabor functions was developed and parameters of Lorentzian line-shape profile and its asymmetry resulting from simultaneous frequency and amplitude response of the current-modulated semiconductor laser were determined. Nonlinear least-square fitting procedure employing differential evolution algorithm was successfully utilized for performing this task. Line-shape fitting procedure enabling efficient signal de-noising and background subtraction of wavelength modulation spectra was implemented into an open-source code.

Keywords: gas sensing, spectroscopy, modulation, model, line-shape asymmetry.

\section{INTRODUCTION}

Tunable Diode Laser Absorption Spectroscopy (TDLAS) has long been recognized as a well-established optical diagnostic tool for gas sensing in various environments (see e.g., [1] and references therein). In order to reduce low-frequency noise inherent to direct TDLAS method, wavelength modulation spectroscopy (WMS) [2] is usually preferred for measurement of low absorption signals.

For analytical purposes it is always desirable to evaluate line-shape profile $[3,4,5,6]$, rather then simply estimate peak-to-peak parameters of WMS signal, which contains less-robust information on molecular absorption and spectral broadening. Therefore, line-shape fitting procedure needs to be addressed in WMS post-processing scheme as the level of uncertainties is a critical attribute of measurement outputs.

Efficient signal de-noising and background subtraction is a serious problem when detecting low-level absorption signals. Spectral coincidence of different absorption features with molecular fingerprints of interest is another issue to be solved for successful interpretation of WMS experiments.

Here we report an automated procedure which was developed in order to address these problems without the need for prior spectral calibration of a particular WMS setup. The solution is based on a novel analytical model of spectral lineshapes relevant to WMS signals from absorbing molecules, which may be present on an optical path of the respective laser beam.

\section{Subject \& Methods}

In a typical WMS setup, signal from the optical detector is demodulated via analog or digital lock-in amplifier at the $n$-th (which is often the second, i.e. $2 f$ ) harmonic frequency of a current-modulated semiconductor laser radiation. The simplest experimental configuration for $2 f$-WMS consists of a single optical path, which can include both probed volume (with an analyte of interest) and a reference cell or gaseous flow suitable for spectral calibration purposes.

The procedure, which is schematically described in Fig. 1. was developed as a first step of our aim to perform quantitative spectroscopy and species concentration measurements in the given experimental setup. Gaseous flow of carbon dioxide $\left(\mathrm{CO}_{2}\right)$ at room temperature was utilized as a feasible reference for spectral calibration. Lorentzian spectral line profile is therefore assumed to be appropriate for the given study. 


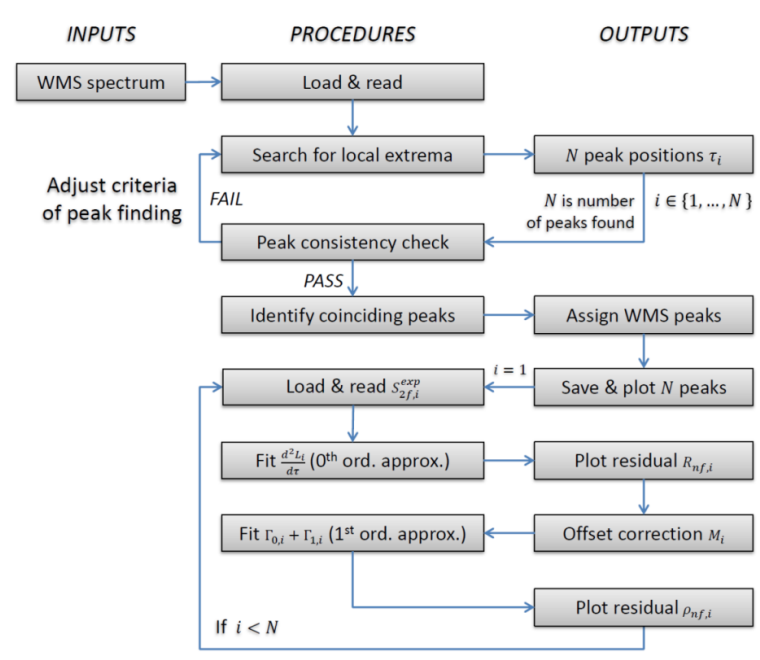

Fig. 1. Procedural steps involved in fitting of new line-shape asymmetry model.

Due to this assumption, applicability of the method reported here is rather limited to experimental conditions when collisional broadening dominates over the Doppler broadening and other physical effects on spectral line shape.

Spectral line shapes were investigated primarily because they contain crucial information for development of proper data processing methodology as well as for optimizing the overall performance of our experimental setup. Theoretical model which we used for the given purpose enabled to efficiently describe the characteristic profile of $2 f$-WMS absorption signal.

Compared with more sophisticated line-shape fittings methods, no further data on experimental setup (modulation intensity, optical power change, etc.) or parameters of spectral line position and width were required as model inputs. Thus, we anticipate that the individual harmonic components of $2 f$-WMS signal can be estimated prior to spectral calibration and determination of wavelength modulation response function for the given semiconductor laser.

Finally, an automated line-shape fitting procedure has been implemented in the Python programming language and its open-source release in the form of interactive script is in preparation.

\subsection{Experimental setup}

Measurements reported here were performed under atmospheric pressure using the body of the burner designed according to [7]. Two separate mass-flow controllers (Manufacturer: Bronkhorst High-Tech B.V.) were employed for feeding the dryed air from a compressor (Manufacturer: JUNAIR/Gast Group Ltd.) into the central body of the burner and carbon dioxide (Manufacturer: Air Liquide Deutschland $\mathrm{GmbH}$, purity $99.995 \%$ ) to annular co-flow at volumetric flow rates of $Q_{v, \text { air }}=10 \mathrm{l} / \mathrm{min}$ and $Q_{v, \mathrm{CO}_{2}}=11 \mathrm{l} / \mathrm{min}$, respectively.

Tuning range of distributed feedback (DFB) laser operating around $1.573 \mu \mathrm{m}$ (Manufacturer: Eblana Photonics Ltd.) was periodically (each $10 \mathrm{~s}$ ) scanned by ramping up electric current at constant diode temperature maintained by laser driver/controller (Manufacturer: Thorlabs Inc.).

Sine wave $(f=11 \mathrm{kHz})$ was superimposed electronically on repeating saw-tooth wave to modulate the lasing wavelength. Signal from the amplified InGaAs photodetector (Manufacturer: Thorlabs Inc.) was demodulated at second harmonic $(2 f)$ frequency by analog lock-in amplifier (Manufacturer: Stanford Research Systems Inc.) and acquired on digital oscilloscope (Manufacturer: Teledyne LeCroy GmbH) at the sampling rate of $1 \mathrm{kS} / \mathrm{s}$.

Finally, accumulation and averaging procedure (each 5 samples) was performed to increase the signal-to-noise ratio (SNR), thus single measurement (averaged scan) was obtained during a $50 \mathrm{~s}$ interval. Optical path length of the laser beam through absorbing medium $\left(\mathrm{CO}_{2}\right.$ coflow stream) was increased by one reflection on the planar mirror, thus yielding $l_{p}=10 \mathrm{~cm}$.

\subsection{Theoretical model}

Spectrally-broadened absorption line shape in an atmospheric-pressure gaseous flow can be approximated by area-normalized Lorentzian profile given by the function:

$$
L=\frac{a_{0}}{\pi a_{2}\left(1+\left(\frac{\tau-a_{1}}{a_{2}}\right)^{2}\right)},
$$

where $a_{0}, a_{1}$, and $a_{2}$ are height (absolute maximum), center and the half-width at half maximum (HWHM) of the Lorentzian function $L$, respectively. Spectral profile is characterized here in temporal domain (by $\tau$ ranging from $\tau=0$ $\mathrm{s}$ to $\tau_{S}=10 \mathrm{~s}$ in our specific case) which is proportional to ramping laser current.

Second derivative of $L$ provides zeroth-order approximation of the $2 f-\mathrm{WMS}$ signal $S_{2 f}$ in case of pure frequency modulation (FM) leading to fully symmetric spectral line shape. Analytical form obtained after symbolic derivation and simplification is given by:

$$
\frac{d^{2} L}{d \tau}=-\frac{2 a_{0} a_{2}\left(a_{2}^{2}-3\left(a_{1}-\tau\right)^{2}\right)}{\pi\left(a_{2}^{2}+\left(a_{1}-\tau\right)^{2}\right)^{3}} .
$$

Fourier series expansion has been previously employed [2] to derive $2 f-\mathrm{WMS}$ analytical expression of line-shape function and its asymmetry in a frequency domain.

However, unlike previous investigators $[3,4,6]$, we report here an alternative use of trigonometric series postulated by Gabor [8] for representation of an arbitrary elementary signal.

Following notation given in (1), Gabor functions were reformulated into general form (for $k=0,1,2, \ldots, \infty$ ):

$$
\begin{gathered}
\Gamma_{k, \cos }=\alpha_{k} \cos \left(2 \pi k \frac{\left(\tau-a_{1}\right)}{2 a_{2}}+\phi_{k}\right) G_{w}, \\
\Gamma_{k, \sin }=\beta_{k} \sin \left(2 \pi\left(k+\frac{1}{2}\right) \frac{\left(\tau-a_{1}\right)}{2 a_{2}}+\psi_{k}\right) G_{w},
\end{gathered}
$$




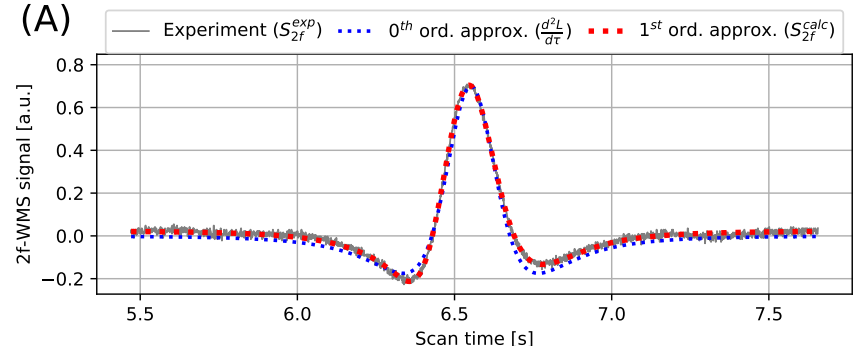

(B)

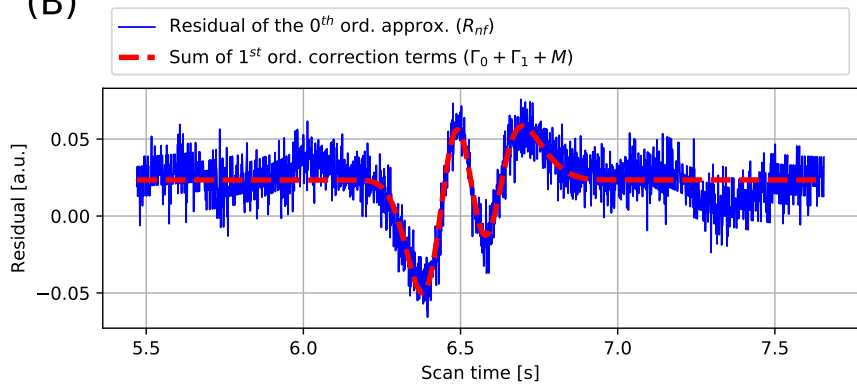

(C) - Residual of the $1^{\text {st }}$ ord. approx. $\left(\rho_{n f}\right) \quad$ - G Gabor function $\left(\Gamma_{1, \cos }\right.$ term) - Gabor function $\left(\Gamma_{0, \text { sin }}\right.$ term $) \quad$ - Gabor function $\left(\Gamma_{1, \sin }\right.$ term $)$

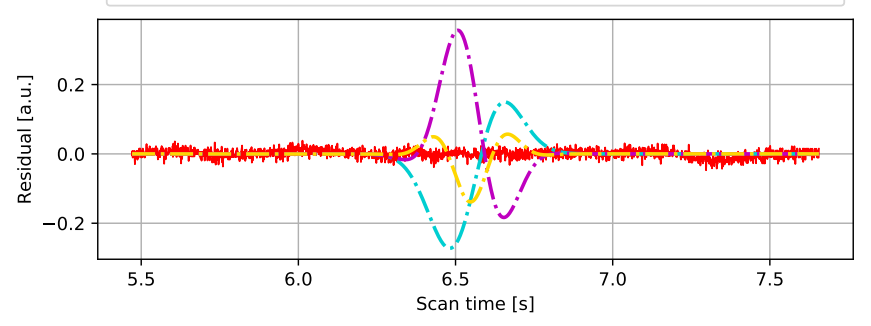

Fig. 2. Spectral profile of $\mathrm{R}(16)$ absorption line of carbon dioxide $\left(2 v_{1}+2 v_{2}+1 v_{3}\right.$ band $)$ in temporal domain and results of line-shape fitting procedure. Experimental data recorded at lock-in phase shift $\Phi=-45^{\circ}$ (with $30 \mathrm{~ms}$ integration time, $200 \mathrm{mV}$ sensitivity and $100 \times$ signal expansion).

$$
\sum_{k=0}^{\infty} \Gamma_{k}=\sum_{k=0}^{\infty}\left(\Gamma_{k, \cos }+\Gamma_{k, \text { sin }}\right) .
$$

Here $\alpha_{k}, \beta_{k}, \phi_{k}$ and $\psi_{k}$ are amplitudes and phase shifts, respectively, of the corresponding term (Gabor function) and $G_{w}$ is a width-adjusted (reduced) Gaussian distribution function described below in more details, see (6).

Only few terms of this expansion (for $k=0$ and $k=1$ ) were considered as essential for our application. To further limit the number of free parameters for least-square fitting procedure, $\Gamma_{0, c o s}$ term was constrained to zero (by assuming $\phi_{0}=\frac{\pi}{2}$ ). In spite of the fact, that $2 f-\mathrm{WMS}$ is in principle zero background technique, significant offset value was observed and thus had to be included into our model following experimental trials. The offset value $M$ was determined as an arithmetic mean of the signal in an appropriate section of the spectrum.

$$
G_{w}=\frac{a_{0}}{w \sigma_{i} \sqrt{2 \pi}} \exp \left[-\left(\frac{\tau-a_{1}}{2 w \sigma_{i}}\right)^{2}\right],
$$

where parameters $a_{0}$ and $a_{1}$ are height (absolute maximum) and center of the respective Lorentzian function specified ac- cording to (1). Initial value of standard deviation $\sigma_{i}$ was determined from $a_{2}$ which is proportional to $\sigma_{i} \sqrt{2 \ln (2)}$. Widthreduction parameter $w$ of the $G_{w}$ envelope was adjusted in order to obtain best-fit representation of the residual signal $\left(R_{n f}=S_{2 f}^{\exp }-\frac{d^{2} L}{d \tau}\right)$ by minimal set of Gabor functions. Effective parameters of three Gabor functions $\left(\Gamma_{0, \sin }\right.$ and both terms of $\Gamma_{1}$ ) were evaluated numerically by nonlinear leastsquare regression (minimization) method yielding:

$$
\rho_{n f}=R_{n f}-M-\Gamma_{0}-\Gamma_{1} \approx 0 .
$$

First-order approximation of the $2 f-\mathrm{WMS}$ signal was thus obtained as:

$$
S_{2 f}^{\exp } \approx S_{2 f}^{\text {calc }}=\frac{d^{2} L}{d \tau}+\Gamma_{0}+\Gamma_{1}+M .
$$

\section{RESULTS}

Measurements were mainly focused on the region around $6360 \pm 2 \mathrm{~cm}^{-1}$ where we observed three spectral lines assigned as $\mathrm{R}(14), \mathrm{R}(16)$ and $\mathrm{R}(18)$ of the $2 v_{1}+2 v_{2}+1 v_{3}$ combination band of $\mathrm{CO}_{2}$ [9]. Experimental data and final results of the theoretical model are depicted in Fig. 2, trace (A).

Apparently, sum of correction terms $\left(M+\Gamma_{0}+\Gamma_{1}\right)$ provides suitable regression function for fitting the $R_{n f}$ residual with an excellent performance in the given case, see trace (B) in Fig. 2. It is worth noting that the width of Gaussian envelope relative to initially estimated $\operatorname{HWHM}\left(a_{2}\right)$ of Lorentzian function had to be reduced (to $w \approx 0.5$ ) in order to achieve appropriate best-fit representation.

Physical interpretation of Gabor function can then be anticipated from the trace (C) in Fig. 2. As $n$ is odd for linecenter asymmetric components of WMS signal, $\Gamma_{0, \sin }$ and $\Gamma_{1, c o s}$ terms are attributable to effect of $1 f$ and $3 f$ modulation, respectively. In analogy, $\Gamma_{1, \sin }$ term resembles contributions from even $n$ (e.g., $4 f$ ) harmonics.

\section{CONCLUSIONS/Discussion}

Based on results summarized in the previous section we presume that the model reproduces some intrinsic features of WMS signal and provides an interesting alternative to nonphysical methods for signal de-noising, e.g., discrete wavelet transformation (DWT) [10]. This new model can also provide initial inputs for physically sound theoretical models, enabling to estimate spectral response and phase-shift parameters (e.g., [3, 6]) from experimental line-shape profiles.

We can conclude that the entire procedure reported in this communication enables to automatically derive analytical description of line-shape asymmetry (i.e., instrumental function) corresponding to the specific experimental setup. Therefore, it has a capability to provide important inputs for simulation of complex absorption spectra when dealing with quantification of concentration or temperature fields in nonhomogeneous gaseous flows (e.g., in laminar flames) based on the $2 f$-WMS technique. 
An open-source software implementation of an automated line-shape fitting procedure is convenient for further development or modifications in frame of various researches as well as educational activities. Extension of the model appropriate for fitting the spectral lines with Voigt profile is in progress [11].

\section{ACKNOWLEDGEMENT}

The article is based upon work from COST Action (CM1404), supported by COST (European Cooperation in Science and Technology). The research was funded by the Ministry of Education, Youth and Sport of the Czech Republic (Project No. LTC17071). Financial support from the Czech Science Foundation (Project No. 17-05167S) and from VSB-Technical University of Ostrava (Faculty of Safety Engineering) via the Project No. SP2017/127 is also acknowledged.

\section{REFERENCES}

[1] Hodgkinson, J., Tatam, R. P. (2013). Optical gas sensing: A review. Measurement Science and Technology, 24(1), 012004.

[2] Kluczynski, P., Gustafsson, J., Lindberg, A. M., Axner, O. (2001). Wavelength modulation absorption spectrometry - an extensive scrutiny of the generation of signals. Spectrochimica Acta Part B: Atomic Spectroscopy, 56(8), 1277-1354.

[3] Schilt, S., Thevenaz, L., Robert, P. (2003). Wavelength Modulation Spectroscopy: Combined Frequency and Intensity Laser Modulation. Applied Optics, 42(33), 6728.

[4] Engelbrecht, R. (2004). A compact NIR fiber-optic diode laser spectrometer for $\mathrm{CO}$ and $\mathrm{CO} 2$ :. Spectrochimica Acta Part A: Molecular and Biomolecular Spectroscopy, 60(14), 3291-3298.
[5] Civiš, S., Zelinger, Z., Nevrlý, V., Dorogan, A., Ferus, M., Iakovlev, V., Sirbu, A., Mereuta, A., Caliman, A., Suruceanu, G., Kapon, E. (2014). Near-infrared waferfused vertical-cavity surface-emitting lasers for HF detection. Journal of Quantitative Spectroscopy and Radiative Transfer, 147, 53-59.

[6] Zhao, G., Tan, W., Hou, J., Qiu, X., Ma, W., Li, Z., Dong, L., Zhang, L., Yin, W., Xiao, L., Axner, O., Jia, S. (2016). Calibration-free wavelength-modulation spectroscopy based on a swiftly determined wavelengthmodulation frequency response function of a DFB laser. Optics Express, 24(2), 1723.

[7] Hartung, G., Hult, J., Kaminski, C. F. (2006). A flat flame burner for the calibration of laser thermometry techniques. Measurement Science and Technology, 17(9), 2485-2493.

[8] Gabor, D. (1946). Theory of communication. Part 1: The analysis of information. Journal of the Institution of Electrical Engineers - Part III: Radio and Communication Engineering, 93(26), 429-441.

[9] Henningsen, J., Simonsen, H. (2000). The (2201-0000) Band of CO2 at $6348 \mathrm{~cm}-1$ : Linestrengths, Broadening Parameters, and Pressure Shifts. Journal of Molecular Spectroscopy, 203(1), 16-27.

[10] Li, J., Parchatka, U., Fischer, H. (2012). Applications of wavelet transform to quantum cascade laser spectrometer for atmospheric trace gas measurements. Applied Physics B, 108(4), 951-963.

[11] Nevrlý, V., Bitala, P., Klečka, V., Vašinek, M., Zelinger, Z., Suchanek, J., Dostál, M., Válek, V. (2019). Analysis of wavelength modulation spectra for determination of $\mathrm{OH}$ radical concentration in an atmospheric pressure laminar premixed flames. Journal of Physics: Conference Series. Manuscript in preparation.

Received January 19, 2018. Accepted July 25, 2018. 